\title{
A Spectroscopic Study of RV Tauri Stars in the LMC
}

\author{
Karen R. Pollard ${ }^{1}$, Tom Lloyd Evans ${ }^{2}$
}

Abstract. Preliminary results from a low-resolution spectroscopic survey of RV Tauri stars and Type II Cepheids in the LMC are presented.

RV Tauri stars are luminous, long-period members of the Type II class of Cepheid variables. A recent investigation of these stars in the LMC (Pollard et al. 1997; Alcock et al. 1998) deduced their absolute luminosities which will help us in our study of their other fundamental parameters.

Spectra of a number of the LMC RV Tauri stars and Type II Cepheids were obtained with the 1.9-m reflector at the South African Astronomical Observatory. These classification-resolution spectra have been used to look for the presence of RV Tauri spectral signatures and allow spectroscopic subtypes to be assigned according to the scheme of Preston et al. (1963). These observations allow us to investigate the inter-relationship between members of the different RV Tauri spectroscopic classes in the LMC as well as helping to define much more precise group characteristics. These characteristics will be used to study the properties of the RV Tauri stars in both the Galactic and LMC environments.

A most unusual set of spectra was obtained of the longest period RV Tauri star in the LMC sample (Pollard \& Lloyd Evans 1999). The spectrum of this star (MACHO*04:55:43.2-67:51:10) is similar to those observed in proto-planetary nebulae. This peculiar star appears to combine the main spectral anomaly of the proto-planetary nebulae with RV Tauri-like photometric variability. This star may represent a link between these two types of objects, strengthening the post-AGB interpretation of RV Tauri stars.

\section{References}

Alcock, C., Allsman, R. A., Alves, D. R., et al. 1998, AJ, 115, 1921

Pollard, K. R., Alcock, C., Alves D. R., et al. 1997, in Proc. 12th IAP Astrophys. Coll., Variable Stars and the Astrophysical Returns of Microlensing Surveys, ed. R. Ferlet, J.-P. Maillard, \& B. Raban (Gif-sur-Yvette: Editions Frontières), 219

Pollard, K. R. \& Lloyd Evans, T. 1999, in IAU Symp. 191, Asymptotic Giant Branch Stars, ed. T. Le Bertre, A. Lèbre, \& C. Waelkens (Michigan: ASP ), 459

Preston, G. W., Krzemiński, W., Smak, J., \& Williams, J. A. 1963, ApJ, 137, 401

\footnotetext{
${ }^{1}$ Department of Physics and Astronomy, University of Canterbury, Private Bag 4800, Christchurch 8020, New Zealand

${ }^{2}$ South African Astronomical Observatory, PO Box 9, Observatory 7935, South Africa
} 